\title{
Vimentin and its role as a biomarker in health and disease
}

\author{
(1) Vaibhav Pandita, (1) Vidya Ajila, (1) G. Subhas Babu, (1) Shruthi Hegde, (1) Mohamed Faizal Asan
}

Nitte (Deemed to be University), AB Shetty Memorial Institute of Dental Sciences (ABSMIDS), Department of Oral Medicine and Radiology, Mangalore, India

Date submitted:

15.10.2020

Date accepted:

27.01.2021

Online publication date: 15.12.2021

\section{Corresponding Author:}

Vidya Ajila, MDS, Nitte (Deemed to be University), AB Shetty Memorial Institute of Dental Sciences (ABSMIDS), Department of Oral Medicine and Radiology, Mangalore, India

ajila_v@yahoo.com

ORCID:

orcid.org/0000-0002-5744-9322

Keywords: Vimentin, epithelial mesenchymal transition, metastasis

\begin{abstract}
Vimentin is an intermediate filament protein responsible for maintaining cellular integrity and resistance to stress. It has a widespread distribution in many cells throughout the body where it forms a cytoskeletal framework. Vimentin plays an important role in the regulation of many cellular and tissue functions. It is overexpressed in malignancies, potentially malignant oral disorders and autoimmune conditions like rheumatoid arthritis and Crohn's disease. It is associated with cell surface binding and replication of viruses such as human immunodeficiency virus (HIV), severe acute respiratory syndrome-related Coronavirus, dengue and encephalitis. In HIV, it is associated with the viral infectivity factor which is associated with HIV replication. It can be used as a biomarker for diagnosis and prognosis and has potential as a therapeutic target in many conditions. The present review focuses on the structure, functions, clinical implications and future scope of vimentin in the management of various diseases.
\end{abstract}

\section{Introduction}

Vimentin is an important intermediate filament protein responsible for maintaining the integrity of the cell and its resistance to stress. It is expressed in mesenchymal cells and tumors and is considered as a biomarker for cellular and tissue development. It is found in many cells during initial embryogenesis and is converted to a specific intermediate filament during differentiation. Vimentin is usually present in fibroblasts, chondroblasts, smooth muscle cells, mesothelium, pericytes, melanocytes and endothelial cells (1).

Vimentin has an important role in many physiological functions such as cell adhesion and motility, maintenance of cytoskeletal structure, cell senescence, and wound healing. It is an important marker in many inflammatory conditions, potentially malignant disorders and autoimmune diseases. In malignancies, it is associated with invasion and metastasis and may have a role in prognosis. It can act as a receptor or co-receptor for many viruses, such as human immunodeficiency virus (HIV), Severe acute respiratory syndrome-related Coronavirus (SARSCoV), dengue and encephalitis viruses, due to its implications in attachment and viral entry into cells (2).

Thus, vimentin is associated with both normal physiological functions as well as pathological conditions. The present review aims to give an overview of this biomarker which has effects on a wide range of cells and tissues and to discuss its scope in diagnosis, prognosis and therapy.

We conducted an online search in "PubMed" using the keywords "Vimentin", "Metastasis", "Epithelial Mesenchymal Transition (EMT)", "Carcinoma" and "Potentially Malignant Disorder". Only manuscripts which had full text available were evaluated and referred for the present narrative review. 


\section{History}

Intermediate filaments were discovered by Buckley and Porter (3) in the late 1960's as a result of advances in microscopic techniques. Franke et al. (4), in 1978, created antibodies to a protein found in the cytoskeleton of mouse 3T3 cells and proposed the name 'vimentin' to differentiate it from proteins in other intermediate filaments. The name 'vimentin' is derived from the Latin word 'vimentum', used to describe arrays of flexible rods. The authors have discovered that vimentin forms a radial arrangement throughout the cytoplasm, more towards the nucleus, and is present in cells derived from mesenchyme.

\section{Structure}

Classified as a type 3 intermediate filament, vimentin structure includes a 310-amino-acid-long a-helical rod having acidic and basic amino acids (5). It consists of $\mathrm{N}$ and $\mathrm{C}$ terminals including amino acids which are hydrophobic in nature (6). The long a-helical rod is acidic in nature while the $\mathrm{N}$-terminal domain, called head domain, is basic in nature due to the presence of 12 arginine residues in its amino acid sequence (7).

\section{Location}

In humans, vimentin is expressed in tissues like skin, lungs, kidney, bone marrow and lymph nodes (4). It is present in cells like precursor cells of the pancreas and nerves, Sertoli cells, fibroblasts, endothelial cells, renal tubular and stromal cells, macrophages, neutrophils, mesangial cells and leukocytes (8). Vimentin forms a network which extends throughout the cell cytoplasm and varies among different cells (9). Non-dividing cells show a uniform distribution while reorganization towards the nucleus occurs on exposure to factors like platelet derived growth factor, oncogenes or few viruses (10).

\section{Functions}

\section{Epithelial to Mesenchymal Transition}

EMT is the process of cellular reprogramming where epithelial cells exhibit mesenchymal phenotype with altered shape and increased motility (6). During EMT, epithelial cells are subjected to various functional and behavioral changes that lead to their differentiation into mesenchymal cells (11). These changes are needed for functions such as embryonic development, homeostasis, and tissue healing as well as in cancer metastases.

The various changes that occur during this phase include loss of epithelial cell junctions, reversal of polarity, alterations in cell shape and cytoskeletal reorganization (12). Increased expression of vimentin is an important marker for EMT as vimentin is a major protein in mesenchymal cells and its expression causes cells to become flat and elongated in shape (13-15). Vimentin also interacts with microtubules and associated motor proteins that lead to cell motility (16). The reverse of EMT is mesenchymal epithelial transition, in which cells develop a mesenchymal- to- epithelial conversion with lower motility and decreased vimentin (8). It has been suggested that increased vimentin may be due to activation of nuclear factor kappa B (nuclear factor kappa light chain enhancer of activated B cells), another regulator of the EMT process, in cancer cells (8).

\section{Cell Proliferation, Differentiation and Apoptosis}

Oncogene expression, which is responsible for higher cellular proliferation, is related to increased amounts of mRNA, protein and the soluble forms of vimentin (17). Cell apoptosis is also dependent upon vimentin since its proteolytic breakdown into a pro-apoptotic variant can trigger apoptosis (18).

\section{Association with Various Oncogenes and Proto- oncogenes}

The structural resemblance of vimentin with certain protooncogenes like c-fos and c-jun and oncogenes like Raf and $\mathrm{v}$-mos has shown that gene expression controlled by vimentin can lead to conversion of normal cells into malignant cells (19). Thus, it plays an important role in cell proliferation (20).

\section{Cell Motility and Adhesion}

Vimentin has been found to influence the width of lamellipodium, a protein used for pseudopodial migration (21). Vimentin attaches and controls focal adhesion kinase (FAK), which can impart motile property to cells as seen at the advancing front of lung cancer. By recruiting VAV2, a Rac1 guanine nucleotide exchange factor to FAK, vimentin can regulate adhesion of cells (22).

\section{Methods of Vimentin Assessment}

Various methods have been used in vimentin assessment. These include immunohistochemistry, real-time (RT) quantitative RT-polymerase chain reaction (PCR), Western Blot test, confocal immunofluorescence microscopy and computerassisted imaging to detect stained areas of the cell using a microscope with a charge-coupled device color camera.

\section{Clinical Significance}

\section{Carcinoma}

The transformation of epithelial cells into malignant cells causes vimentin upregulation and loss of keratin (23). Immunofluorescence, Western blot and RT-PCR analysis have shown high vimentin expression in hyperplastic and dysplasia tissues. Increased vimentin expression has been detected in sites with known carcinoma and in oral squamous cell carcinoma (OSCC), vimentin expression has been found to increase with the grade of the tumor (24). These findings are due to the role of vimentin in EMT. Vimentin regulates EMT in three ways:

I. Gene expression: Vimentin regulates expression of $A x \mid 3$ which can induce EMT. 
II. Protein-protein interaction: Vimentin stabilizes the cell at a protein level (25) and ensures cell polarization during cell migration.

III. Phosphorylation: Vimentin phosphorylation protects it from proteolysis, thereby enhancing migration and metastasis of cells (26-28).

Increase in vimentin expression has been noted in cancers of the prostate, breast, endometrium, central nervous system, gastrointestinal tract as well as malignant melanoma, in which it is associated with both diagnosis and prognosis. Vimentin has been used as a diagnostic marker for colorectal carcinoma (8).

Li et al. (29) found increased expression of vimentin in primary malignant melanoma with hematogenous route of metastasis as compared to those without hematogenous metastasis. Vimentin is important for EMT which, in turn, is important for migration and invasion characteristics of cells.

\section{Metastatic Spread}

Vimentin is associated with tumor invasion. Studies have shown that highly invasive breast carcinoma cells without vimentin are more pliant and have decreased tendency to metastasize $(8,23)$. Loss of vimentin significantly has decreased the migration and invasion of cells in dense cultures while vimentin expression has been associated with longer persistence of migration (30). Vimentin is the protein most implicated in metastasis in OSCC. Increased vimentin expression is also associated with increased lymph node metastasis. Bhardwaj et al. (31) investigated vimentin in eyelid sebaceous gland carcinoma and found that overexpression was associated with lymph node metastasis and poor survival.

A study on lung adenocarcinoma found that vimentin was not associated with the development of primary tumors. However, loss of vimentin resulted in a $50 \%$ decrease in lymph node metastasis (23).

\section{Leukoplakia}

Cytoplasmic immunostaining for vimentin demonstrated higher vimentin expression for non-homogeneous leukoplakia when compared to homogenous leukoplakia where it was confined to basal and suprabasal layers of oral mucosa (24).

In leukoplakia, there is decreased expression of epithelial junctions leading to partial or total loss of cell polarity (32). This is a part of EMT, which in turn is regulated by vimentin; hence vimentin expression increases in leukoplakia.

\section{Oral Submucous Fibrosis}

Oral submucous fibrosis (OSMF) is a potentially malignant disorder affecting the oral cavity and sometimes the pharynx. Cases show weak to moderate expression of vimentin, which is confined to the suprabasal layers of oral mucosa in majority of the cases (24).
In OSMF, arecoline triggers the formation of collagen, and exhibits cytotoxicity, change in cell morphology and DNA synthesis (33). This, in turn, causes interference in cellular mitosis and transport mechanisms within the cell. Since vimentin has an important role in cell cytoskeleton, disruption of cytoskeleton in OSMF causes increased vimentin expression $(34,35)$.

\section{Rheumatoid Arthritis}

Vimentin is a type of antigen found in synovial tissue and fluid. In rheumatoid arthritis (RA), vimentin citrullination and presentation to $T$ cells causes formation of anti-citrullinated protein antibody, which can be demonstrated in the synovial fluid (36). This antigen-antibody reaction promotes inflammation which further aggravates the disease process (37).

\section{Crohn's Disease}

Crohn's disease is a genetically mediated inflammatory bowel disease of the gastrointestinal tract associated with increased vimentin levels. Studies have shown that the inflammatory tissue damage and the resulting intestinal fibrosis may be a result of EMT. The areas with fibrosis exhibit EMT markers like vimentin implying a role for EMT in the pathophysiology of Crohn's disease (27).

\section{COVID-19}

Vimentin is associated with viral fusion and replication and also enhances cell surface binding and viral entry. This is true for all viruses except human papilloma virus, in which it has the reverse effect. Studies have shown that interference in expression of vimentin or cell treatment using anti-vimentin antibodies can decrease few viral infections. Cell entry of SARS-CoV may be mediated through vimentin. As SARSCoV and SARS-CoV-2 have similar spike protein sequences, vimentin may be a co-receptor for both. It may also decrease the immune response to the virus and decrease its lethal effects through its effects on the cytokine storm syndrome. Vimentin present on the surface of platelets and endothelial cells acts as a receptor for von Willebrand factor facilitating platelets to bind to subendothelial collagen and causing intravascular thrombin generation and microthrombus formation. Drugs effective against viral infection are also known to cause a decrease in vimentin. Since vimentin is involved in viral infection and lung inflammation, drugs targeting vimentin may be effective in the treatment of COVID-19. Decreased vimentin at cell surface would cause decreased viral binding, decreased intracellular vimentin would decrease viral replication and less vimentin in inflammatory cells would cause decreased lung inflammation. Melatonin, niclosamide, endogenous hydrogen sulfide and simvastatin are a few of the medications that have been suggested to decrease lung injury through their effects on vimentin $(2,38)$. 


\section{Anti-HIV Therapy}

Vimentin expressed in monocytes is important for maintaining cell structure. Since vimentin is broken down by HIV-I proteases, vimentin is postulated to have a role in HIV infection. Antivimentin antibody has been associated with inhibition of virus production by macrophages. It was also reported that viral infectivity factor, which is linked with the processes of viral RNA folding and packaging, had a spatial overlap with vimentin and could collapse the vimentin framework (28,39-41). Modification of endogenous vimentin levels inhibited HIV replication.

\section{Wound Healing}

Wound and tissue healing needs cell motility and adhesion. Vimentin causes an increase in fibroblast and collagen formation and helps in epithelialization of wounds (26). Vimentin acts by influencing the actin-myosin machinery, which is an important factor for wound healing but its exact mechanism of action has not yet been elucidated (26).

\section{Atherosclerosis}

Diseases of the cardiovascular system mayalso be associated with increased vimentin levels. Cells of the cardiovascular system have the capacity to differentiate into mesenchymal-like cells in a manner similar to the EMT observed with increased vimentin levels. Atherosclerosis has been associated with increased vimentin due to EMT. Vimentin null mice have been associated with arterial stiffness and endothelial abnormalities (42).

\section{Cataracts}

Clouding of the lens of the eye is termed as cataract. Normally, the epithelial cells in the lens show a low expression of vimentin. Cataracts are associated with misfolding and increased expression of vimentin. This increase in vimentin may be due to mesenchymal transformation of the epithelial cells of the lens. Although all cases of cataract may not be a result of EMT, EMT may be one of the causes of lens opacification. EMT suppression may thus have potential in the treatment of cataracts (20).

\section{Aging}

Vimentin has been implicated in cell senescence. Senescent cells have an increase in vimentin levels and secrete an oxidized form of vimentin. The glycated form of vimentin is seen in skin fibroblasts and is implicated as a sign in skin aging (20).

\section{Ongoing Research}

Vimentin is an important subject of ongoing research with 1,684 publications in 'PubMed' within the last one year. Recent research has focused on vimentin as a prognostic marker in various cancers, its role in tumor invasion, metastasis and as a potential target in cancer treatment (23). Anti-vimentin antibodies have been implicated in kidney transplant rejection (43). Its scope as a therapeutic target in viral infections is also being investigated (2). Most recently, vimentin has been proposed as a target in the treatment of COVID-19 (38).

\section{Future Scope}

Vimentin is an important protein in cancer metastasis. Drugs aiming at targeting vimentin could decrease cancer metastasis, thereby decreasing cancer related mortality. A multitude of factors are responsible for regulating vimentin expression in tissues. A valid drug trial therefore requires further research into the factors responsible for gene expression of vimentin (10). Due to its widespread expression in normal and abnormal tissues, it is an attractive potential therapeutic target for many disease conditions.

\section{Conclusion}

Vimentin is a crucial biomarker for the detection of many conditions with a wide range of biological functions. It is associated with increased growth, invasion and migration in cancer cells. It is used as a biomarker in both diagnosis and prognosis in various cancers. Further, overexpression of vimentin is also associated with other conditions like RA, potentially malignant oral disorders and delay in wound healing. The main process through which it regulates the above functions is through EMT. Various factors are associated with the functional diversity of vimentin and if we understand the association between various quantifiable biological phenomena, it will increase treatment options. Vimentin has potential as a drug target for many diseases. However, further research into factors affecting vimentin expression is essential prior to drug trials.

\section{Ethics}

Peer-review: Externally peer-reviewed.

\section{Authorship Contributions}

Concept: V.P., V.A., Design: V.A., S.H., M.F.A., Data Collection or Processing: V.P., Analysis or Interpretation: G.S.B., Literature Search: V.P., V.A., M.F.A., Writing: V.P., V.A., G.S.B., S.H., M.F.A.

Conflict of Interest: No conflict of interest was declared by the authors.

Financial Disclosure: The authors declared that this study received no financial support.

\section{References}

1. Damjanov I. Antibodies to intermediate filaments and histogenesis. Lab Invest. 1982;47:215-217.

2. Ramos I, Stamatakis K, Oeste CL, Pérez-Sala D. Vimentin as a Multifaceted Player and Potential Therapeutic Target in Viral Infections. Int J Mol Sci. 2020;21:4675. 
3. Buckley IK, Porter KR. Cytoplasmic fibrils in living cultured cells. A light and electron microscope study. Protoplasma. 1967;64:349-380.

4. Franke WW, Schmid E, Osborn M, Weber K. Different intermediate-sized filaments distinguished by immunofluorescence microscopy. Proc Natl Acad Sci U S A. 1978;75:5034-5038.

5. Perreau J, Lilienbaum A, Vasseur M, Paulin D. Nucleotide sequence of the human vimentin gene and regulation of its transcription in tissues and cultured cells. Gene. 1988;62:716.

6. Parry DA. Hendecad repeat in segment $2 A$ and linker L2 of intermediate filament chains implies the possibility of a righthanded coiled-coil structure. J Struct Biol. 2006;155:370374.

7. Herrmann $\mathrm{H}$, Aebi U. Intermediate filaments: molecular structure, assembly mechanism, and integration into functionally distinct intracellular Scaffolds. Annu Rev Biochem. 2004;73:749-789.

8. Satelli A, Li S. Vimentin in cancer and its potential as a molecular target for cancer therapy. Cell Mol Life Sci. 2011;68:3033-3046.

9. Valgeirsdóttir S, Claesson-Welsh L, Bongcam-Rudloff E, Hellman U, Westermark B, Heldin CH. PDGF induces reorganization of vimentin filaments. J Cell Sci. 1998;111:1973-1980.

10. Inagaki M, Gonda Y, Ando S, Kitamura S, Nishi Y, Sato C. Regulation of assembly-disassembly of intermediate filaments in vitro. Cell Struct Funct. 1989;14:279-286.

11. Kalluri R, Neilson EG. Epithelial-mesenchymal transition and its implications for fibrosis. J Clin Invest. 2003;112:17761784.

12. Kalluri R, Weinberg RA. The basics of epithelialmesenchymal transition. J Clin Invest. 2009;119:14201428.

13. Ivaska J. Vimentin: Central hub in EMT induction? Small GTPases. 2011;2:51-53.

14. Gilles C, Polette M, Zahm JM, Tournier JM, Volders L, Foidart JM, et al. Vimentin contributes to human mammary epithelial cell migration. J Cell Sci. 1999;112:4615-4625.

15. Mendez MG, Kojima S, Goldman RD. Vimentin induces changes in cell shape, motility, and adhesion during the epithelial to mesenchymal transition. FASEB J. 2010;24:1838-1851.

16. Liao G, Gundersen GG. Kinesin is a candidate for crossbridging microtubules and intermediate filaments. Selective binding of kinesin to detyrosinated tubulin and vimentin. J Biol Chem. 1998;273:9797-9803.

17. Rathje LS, Nordgren N, Pettersson T, et al. Oncogenes induce a vimentin filament collapse mediated by HDAC6 that is linked to cell stiffness. Proc Natl Acad Sci U S A. 2014;111:1515-1520.
18. Olson EN, Capetanaki YG. Developmental regulation of intermediate filament and actin mRNAs during myogenesis is disrupted by oncogenic ras genes. Oncogene. 1989;4:907-913.

19. Chen M, Puschmann TB, Marasek P, et al. Increased Neuronal Differentiation of Neural Progenitor Cells Derived from Phosphovimentin-Deficient Mice. Mol Neurobiol. 2018;55:5478-5489.

20. Danielsson $F$, Peterson $M K$, Caldeira Araújo $H$, Lautenschläger F, Gad AKB. Vimentin Diversity in Health and Disease. Cells. 2018;7:147.

21. Helfand BT, Mendez MG, Murthy SN, et al. Vimentin organization modulates the formation of lamellipodia. Mol Biol Cell. 2011;22:1274-1289.

22. Havel LS, Kline ER, Salgueiro AM, Marcus Al. Vimentin regulates lung cancer cell adhesion through a VAV2Rac1 pathway to control focal adhesion kinase activity. Oncogene. 2015;34:1979-1990.

23. Strouhalova K, Přechová M, Gandalovičová A, Brábek J, Gregor M, Rosel D. Vimentin Intermediate Filaments as Potential Target for Cancer Treatment. Cancers (Basel). 2020;12:184

24. Sawant SS, Vaidya Mm, Chaukar DA, et al. Clinical significance of aberrant vimentin expression in oral premalignant lesions and carcinomas. Oral Dis. 2014;20:453-465.

25. Phua DC, Humbert PO, Hunziker W. Vimentin regulates scribble activity by protecting it from proteasomal degradation. Mol Biol Cell. 2009;20:2841-2855.

26. Burgstaller G, Gregor M, Winter L, Wiche G. Keeping the vimentin network under control: cell-matrix adhesionassociated plectin $1 \mathrm{f}$ affects cell shape and polarity of fibroblasts. Mol Biol Cell. 2010;21:3362-3375.

27. Kreis S, Schönfeld HJ, Melchior C, Steiner B, Kieffer N. The intermediate filament protein vimentin binds specifically to a recombinant integrin alpha2/beta1 cytoplasmic tail complex and co-localizes with native alpha2/beta1 in endothelial cell focal adhesions. Exp Cell Res. 2005;305:110-121.

28. Zhu QS, Rosenblatt $\mathrm{K}$, Huang $\mathrm{KL}$, et al. Vimentin is a novel AKT1 target mediating motility and invasion. Oncogene. 2011;30:457-470.

29. Li M, Zhang B, Sun B, et al. A novel function for vimentin: the potential biomarker for predicting melanoma hematogenous metastasis. J Exp Clin Cancer Res. 2010;29:109.

30. Battaglia RA, Delic S, Herrmann H, Snider NT. Vimentin on the move: new developments in cell migration. F1000Res. 2018;7:F1000 Faculty Rev-1796.

31. Bhardwaj M, Sen S, Chosdol K, et al. Vimentin overexpression as a novel poor prognostic biomarker in eyelid sebaceous gland carcinoma. $\mathrm{Br} \mathrm{J}$ Ophthalmol. 2020;104:879-884.

32. Caldeira PC, Abreu MH, do Carmo MA. Binary system of grading oral epithelial dysplasia: evidence of a bearing to 
the scores of an immunohistochemical study. J Oral Pathol Med. 2012;41:452-453.

33. Shah N, Sharma PP. Role of chewing and smoking habits in the etiology of oral submucous fibrosis (OSF): a casecontrol study. J Oral Pathol Med. 1998;27:475-479.

34. Enzinger FM, Weiss SW. Soft Tissue Tumors. 3rd edition. St. Louis, Mo, USA: Mosby; 1995

35. Ross MH, Kaye GI, Pawlina W. Histology-A Text and Atlas. 4th edition. Philadelphia, Pa, USA: Lippincott Williams \& Wilkins; 2003.

36. Van Steendam K, Tilleman K, De Ceuleneer M, De Keyser F, Elewaut D, Deforce D. Citrullinated vimentin as an important antigen in immune complexes from synovial fluid of rheumatoid arthritis patients with antibodies against citrullinated proteins. Arthritis Res Ther. 2010;12:R132.

37. van Venrooij WJ, Pruijn GJ. An important step towards completing the rheumatoid arthritis cycle. Arthritis Res Ther. 2008;10:117.

38. Li Z, Paulin D, Lacolley P, Coletti D, Agbulut O. Vimentin as a target for the treatment of COVID-19. BMJ Open Respir Res. 2020;7:e000623.
39. Zhang H, Pomerantz RJ, Dornadula G, Sun Y. Human immunodeficiency virus type 1 Vif protein is an integral component of an mRNP complex of viral RNA and could be involved in the viral RNA folding and packaging process. J Virol. 2000;74:8252-8261.

40. Karczewski MK, Strebel K. Cytoskeleton association and virion incorporation of the human immunodeficiency virus type 1 Vif protein. J Virol. 1996;70:494-507.

41. Fernández-Ortega $C$, Ramírez A, Casillas D, et al. Identification of Vimentin as a Potential Therapeutic Target against HIV Infection. Viruses. 2016;8:98.

42. Langlois B, Belozertseva E, Parlakian A, et al. Vimentin knockout results in increased expression of sub-endothelial basement membrane components and carotid stiffness in mice. Sci Rep. 2017;7:11628.

43. Rampersad C, Shaw J, Gibson IW, et al. Early AntibodyMediated Kidney Transplant Rejection Associated With Anti-Vimentin Antibodies: A Case Report. Am J Kidney Dis. 2020;75:138-143. 